\title{
Fancy a Drink in Canary Wharf?: A User Study on Location-Based Mobile Search
}

\author{
Alia Amin ${ }^{1, *}$, Sian Townsend ${ }^{2}$, Jacco van Ossenbruggen $^{1,3}$, and Lynda Hardman ${ }^{1,4}$ \\ ${ }^{1}$ Centrum Wiskunde \& Informatica, Amsterdam, The Netherlands \\ ${ }^{2}$ Google UK Ltd., London, United Kingdom \\ ${ }^{3}$ VU University, Amsterdam, The Netherlands \\ ${ }^{4}$ University of Amsterdam, The Netherlands
}

\begin{abstract}
We present a web-based diary study on location-based search behavior using a mobile search engine. To capture users' location-based search behavior in a ubiquitous setting, we use a web-based diary tool that collects users' detailed mobile search activity, their location and diary entries. This method enables us to capture users' explicit behavior (query made), their implicit intention (motivation behind search) and the context (spatial, temporal, and social) in which the search was carried out. The results of the study show that people tend to stick closely to regularly used routes and regularly visited places, e.g. home and work. We also found that most location-based searches are conducted while in the presence of others. We summarize our findings and offer suggestions to improve location-based search by using features such as location-based service mash-ups.
\end{abstract}

Keywords: Location-based search, local search, mobile search, diary study.

\section{Introduction}

While the World Wide Web allows access to information globally, local geographical aspects are nonetheless important in many web search tasks. In a generic search, such as searching for a movie trailer or a book, geographical aspects are irrelevant. This is not the case for location-based searches. For example, when a user is searching for the nearest pubs, the system is required to identify local pubs and only present those that are in the neighborhood. Unlike generic search, the geographical context is important for location-based search. Much research has shown consistent demand for locationbased information on the desktop as well as on mobile devices [9,21,22]. Estimates on how often such search occurs have been disclosed by several major search engines: in [21], samples of queries from 2001 Excite desktop searches were examined and $19.7 \%$ of them were searching for places, people and things; around $9-10 \%$ of the queries collected in [22] on Yahoo! mobile search were identified to have geographical search intentions, whereas more than $15 \%$ of 1 million Google queries from PDA devices are for local services [9]. In addition to search engines, many web services offer search support for diverse location-based information, such as, local businesses

\footnotetext{
* This work was conducted while working as an intern at Google UK Ltd., London.
} 
search and review (e.g. www.yelp.com), city guide (e.g. www.citysearch.com), or local traffic news (e.g. www.highways.gov.uk/traffic). These examples show a healthy market for location-based information providers.

Research on location-based information needs is still at an early stage and most reports are confined to the different domains of interest related to location-based search. For example, large scale mobile query analyses done by Google [9], Yahoo! [2] and an EU Mobile operator [5] consistently report that people make location-based queries on a wide range of domains of interests, such as food and drink (e.g. restaurants), shopping (e.g. stores) and travel (e.g. addresses). A small scale diary study on mobile information needs [20] reported that people search for generic information, such as looking for music, and for location-based information, such as looking for places of interest (POI), business hours, and movie times. In these types of searches it might be useful to consider location context as it has proven to improve the quality of query prediction in a mobile search application [10]. Unfortunately, past research on has not given any explanation as to when, where and why people conduct location-based search. Most research on mobile information needs do not focus on this topic e.g $[2,5,9,20]$, and those that do, limit their approach to automatic query log analysis e.g. $[18,19]$. As far as we know, there is no user study that exclusively investigates people's location-based search motivations and context. In order to improve and optimize location-based services, it is necessary to understand people's location-based information needs and the context in which they occur.

We define location-based search as: "Search for a business or place of interest that is tied to a specific geographical location." This definition is somewhat broader than Location-aware search where it is implied that the system has knowledge of and exploits the searcher's location. We use this terminology to reflect the type of query that is collected from the search engine in this study. In this research, we investigate location-based search on mobile devices. The contribution of this paper is an in-depth investigation of location-based search behavior using the mobile search engine and includes the spatial, temporal, and social contexts in which this search occurs. We look into how the users' location and the people they were with influence the location-based search made on a mobile device. We collect comprehensive information from search engine log data, location data tracking and diary entries. Key findings include, first, that people specify location-based search at different levels of granularity, from simple to detailed queries, constructed by different types of information. Second, people tend to travel along regular routes in their environment and visit the same places of interest regularly, and the impacts on their search behavior. Third, most location-based searches on mobile devices are conducted in the company of other people, such as friends, family or colleagues.

This paper is structured as follows. In the next section, we describe our research method. We then present the results, including how people express location-based searches, the contexts in which the searches are conducted, and the search tools used when they do location-based search. This is followed by discussions on the key findings and design considerations to improve location-based services. We close with a summary and future work. 


\section{Research Method}

We want to investigate how people express location-based information needs on a mobile device, what are the different situations when this search occurs and what are the search tools that people use to conduct location-based search. Our research questions are as follows:

What types of location-based search can be identified?

In what context (spatial, temporal, and social) are these searches initiated?

What are the information sources (e.g. maps) used for location-based searches?

Capturing users' behavior on a mobile platform is challenging because of the difficulty of unobtrusively collecting data in a ubiquitous environment. Previous research with the mobile phone relies on different methods, such as interview e.g. [8], log data analysis e.g. [2], (video) observation e.g. [14], experience sampling e.g. [7], diary study e.g. [3,20], or a combination of two or more methods [17]. Search logs provide data on users' realistic search engine usage. They do not, however, provide any insight into users' intentions. Identifying location-based information needs solely from log analysis is not easy because the intention might not always be expressed explicitly in the query by specifying an address or a city name [22]. Diary studies give the opportunity for users to express search goals. Our approach is to combine two methods: search logging and diary study in order to benefit from the strengths of both. Additionally, to understand how people perceive the spatial world around them and the places that are important to them, we use a method similar to that proposed in [13] in the form of a creative exercise where people describe and visualize their environment and the places they go. Next, we describe the web diary tool, the user study procedure and participant profile.

\subsection{The Web-Based Diary Tool}

The diary tool collects and links 3 types of data: users' search event logs from the Google mobile search engine, location tracking data from participant's device and diary entry data.

Search event log. Events occurring using Google mobile search ${ }^{1}$ were collected (e.g., queries entered, clicks, scrolls, keystrokes) including the corresponding timestamps. We also collected SMS snippets. Participants were asked to send an SMS to a dedicated number whenever they identified an information need that was answered by some other information source (e.g. maps, other websites) or a need that could not be answered.

Location tracking. We logged the user's location (latitude and longitude), every time a search is made. The location data provides information on the participants' whereabouts when the search occurs.

Diary entry. Participants were required to log into the diary at the end of every day and to answer questions about their search activity. The participant's detailed search

${ }^{1}$ A software was installed on users' phones to enable us to log search events and location (latitude and longitude) while users were searching with the Google Mobile Search. http://www. google.com/mobile/ 
history throughout the day is made available. There are 2 steps of action in the diary tool. First, participants need to identify search tasks. In some cases, information needs can only be answered by a participant through conducting several queries in a search session. For example, a user might type several queries, such as pubs, bars, Irish pubs, for one search task: to find the nearest pubs. Thus, in the web-diary tool, we provide an option for the participant to group these queries from the same session into a single search task. Second, for every search task, participants need to answer several questions:

\section{How important is this task? \\ Where were you? \\ What were you doing? \\ Who were you with? \\ Describe in detail what you needed to look for. \\ When was this need initiated? \\ What tool(s) did you use to find your answer? \\ Did you successfully accomplish your task? \\ Positive and negative experiences with the tool used?}

The search event log provides information on the location-based information needs that occurred and the search history. The location tracking indicates where the search took place, and the answers provided by the participants from these questions give an overview on the condition and situation when the information need arose.

\subsection{Procedure}

The study is divided into four parts:

Pre-study interview. Participants were briefed about the procedure and were requested to provide background information about their daily activities and search experiences. Participants were free to search for any topic but were told that this study was investigating location-based search. Participants gave their consent to have their search activity logged and location tracking during the course of the study.

Digital diary study. The diary study lasted for 12 days. The duration of the study covered different types of days (6 work days, 6 days weekend/public holidays). During this period, participants were asked to make approximately 3 queries per day, as a loose guideline, with the search client on their phone, and to log into the diary website daily from their PC to answer questions related to the mobile searches made.

SMS Reminder. During the course of the diary study, an SMS was sent every 2 or 3 days as a reminder to make queries, and to $\log$ into the online diary daily.

Post-study interview. After the study, participants were invited for a semi-structured interview. They were asked additional questions relating to their mobile search experiences based on the answers provided on the diary website. Any unclear or missing diary entries were clarified. Finally, participants were given a creative exercise to sketch typical places where they usually go, and to specify the distance and means of travel to get there. 


\section{Participants}

Twelve participants were recruited from the London area from which 9 participants (5 female, 4 male) successfully completed the whole study. We recruited participants who used Blackberry phones ${ }^{2}$. All nine participants were young professionals, aged between 27-35 years old $(M=32.6, S D=2.5)$. We recruited participants with different occupations, such as a financial advisor, photographer, and retail sales person. All participants were regular users of mobile phones and PCs. Our participants use their phones for calls and SMS-es on a regular basis. In addition, they have a mobile Internet subscription and use it to access their emails, news and entertainment content (e.g. BBC), to plan journeys (e.g. maps, public transport schedules), to keep in touch (e.g. Facebook) and to do search (e.g. Google, Yahoo!).

\section{Results}

During the course of the study, we collected 347 location-based mobile search queries (see Table 1). These were organized by participants into 186 location-based search tasks (see Fig. 1B). Thus, in average, participants make 1.87 queries per locationbased search task. Additionally, there were 13 search tasks (6.3\%) which were not location-based search tasks, such as downloading images, music, or games (i.e. Transaction task ${ }^{3}$ ). We omit these and focus our analysis and discussion on locationbased search tasks. The diary tool provides a rich context (who, where, when, why, and how) for each search task conducted. Queries and tasks were systematically analyzed as follows. First, we examine the queries from different perspectives to understand how people express location-based information needs, the different domains of interest and different types of search tasks. Second, we examine the answers provided from each diary entry to understand the spatial, temporal, and social context when location-based information needs arose. Third, we examine the tools that people use to find location-based information and the reasons behind unsuccessful location-based search. Finally, we use affinity diagram technique to understand emerging topics that lead to the discussions and design implications.

\subsection{Types of Location-Based Queries}

The queries were analyzed from 3 different perspectives. Each perspective offers a unique view on location-based search. We examined the query patterns to identify how people express location-based information needs (Syntactic view). Afterwards, we looked at the variety of domains to get an overview of peoples' interests and on the types of places people look for the most (Domain view). Finally, we look from the perspective of information seeking tasks, to understand the breadth of the search goal (Task view).

\footnotetext{
${ }^{2}$ Due to a technical restriction of our system.

${ }^{3}$ Transaction task is an information exchange task, e.g., online banking, downloading multimedia documents. Transactions are often associated with a user name and password combination [12].
} 
Table 1. How people express location-based information needs (Total: 347 queries)

\begin{tabular}{|c|c|c|c|}
\hline & & Query & example \\
\hline & $67.7 \%$ & \multicolumn{2}{|c|}{ Simple location-based query (235 queries) } \\
\hline a. & $22.9 \%$ & Business & $\begin{array}{l}\text { the orange tree pub }[\mathrm{P} 4], \text { Charlie harmer }+ \text { dry cleaning } \\
\text { [P5] }\end{array}$ \\
\hline b. & $4.3 \%$ & Business category & Mexican restaurant $[\mathrm{P} 6]$ \\
\hline c. & $2.4 \%$ & Event & cbeebies + tour $[\mathrm{P} 5]$ \\
\hline d. & $7.2 \%$ & Product/service & smart 12 month lease $[\mathrm{P} 6]$, vintage leather jacket $[\mathrm{P} 7]$ \\
\hline e. & $2.9 \%$ & Location & Where is covent garden $[\mathrm{P} 3]$, pennyhill park $[\mathrm{P} 4]$ \\
\hline f. & $10.1 \%$ & Local news, weather & $B B C$ weather $[\mathrm{P} 1]$ \\
\hline g. & $5.8 \%$ & Transport schedule, map & train times $[\mathrm{P} 1]$, tube map $[\mathrm{P} 4]$ \\
\hline \multirow[t]{2}{*}{ h. } & $12.1 \%$ & Url & Streetmap.co.uk [P4], kidslovelondon.com [P11] \\
\hline & $32.3 \%$ & \multicolumn{2}{|c|}{ Detailed location-based query (112 queries) } \\
\hline i. & $1.4 \%$ & Business and attribute & tesco opening hol \\
\hline j. & $13.0 \%$ & Business and location & burger king charing cross [P6], nandos - se19 [P3] \\
\hline k. & $5.8 \%$ & Business category and location & canary wharf bars [P2], model agencies in London [P7] \\
\hline 1. & $1.9 \%$ & Event and location & moon walk 2008 london [P7] \\
\hline $\mathrm{m}$. & $6.8 \%$ & Product/service and location & $\begin{array}{l}\text { driving testing centre - hither green[P3], paint balling } \\
\text { Victoria[ } \mathrm{P} 11]\end{array}$ \\
\hline n. & $3.4 \%$ & Multiple locations & directions from green park to primrose hill $[\mathrm{P} 7]$ \\
\hline
\end{tabular}

\subsubsection{Syntactic View}

We manually analyzed each query to investigate how people express location-based queries (see Table 1). There are 6 different components of a location-based query: business name (e.g. Orange Tree pub), business category (e.g. Mexican restaurant), location name (e.g. Pennyhill Park), event name (e.g. Cbeebies tour), product or service (e.g. Smart 12 month lease), and web address (e.g. www.streetmap.co.uk). We found that participants express location-based information needs in different levels of granularity with these elements: (a) A simple location-based query consists of only one of these components (see Table 1, a-e). Additionally, some people make Navigational queries ${ }^{4}$ to search for a website that contains location-based information (see Table 1, f-h), e.g. www.streetmap.co.uk, www.tfl.gov.uk/tube, or www.kidslovelondon.com. These sites usually offer dynamic and detailed information, such as tube schedules, the city's weather forecast, and local events. (b) A detailed location-based query consists of a combination of more than one element, e.g. business name and location name (see Table 1, i-n). From Table 1, we observe that location-based information need is expressed by specifying a business name (22.9\%), a business name and location (13.0\%), or a web address containing local information (12.1\%).

\subsubsection{Domain View}

The queries were further analyzed to identify the domains of interest. Four main domains of interest that our participants looked for (see Fig. 1A): stores (27.0\%): businesses that offers products/services, such as electronic stores, furniture stores, book stores; food \& drink (24.5\%): businesses such as restaurants, pubs, cafes; entertainment (13.7\%): such as cinemas, theaters, concerts; and transportation (10.3\%): public or private companies, such as train, bus, tube, taxi.

\footnotetext{
${ }^{4}$ Navigational query is a query where the intention is to reach a particular site [4].
} 


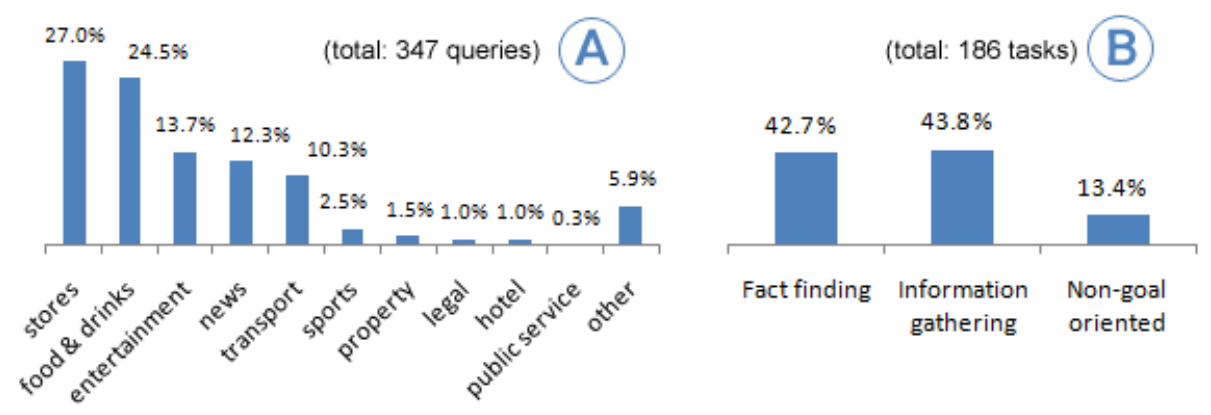

Fig. 1. A) Distribution of domains of interest. B) Types of location-based search task.

\subsubsection{Task View}

The diary entries provide rich descriptions of users' search tasks and motivation behind each search query. We found there are different location-based search tasks (see Fig. 1B) from specific to broad as explained by the Information Seeking Task taxonomy $[1,11,12]$. These tasks are:

(a) Fact Finding tasks. The task is goal oriented and focused: Users look for specific factual pieces of information. The search intention is usually straightforward e.g. looking up a phone number. We found $42.7 \%$ of the location-based search tasks fall into this category. Examples of participant's tasks are: Looking for service information: public transport timetable. "I want to know the train times home to return to London." [P1]. Looking for business information e.g. contact details. "I needed the number for Pizza Express to order the food." [P12]

(b) Information Gathering tasks. Users carry out several search tasks to fulfill a higher level goal. We found $43.8 \%$ of the search tasks belong in this category. Examples of participants' tasks are: making a decision where to go for dinner by exploring different businesses with different constraints, such as cost, distance and product. "Looking for a Mexican restaurant for a surprise birthday party, found a good selection of restaurants with distance information and maps to easily locate." [P6] ."We were looking for a reasonably priced place to go and eat in central London as that would be convenient for everyone." [P11]

From our search logs, we observe that Information Gathering search tasks tend to be iterative (the user goes through several rounds of searches), exploratory (the user tries out different alternative queries/search strategies), and comparative (comparing different search results before making a decision).

(c) Non-goal oriented information seeking tasks. Users' information needs are not goal driven. The motivation of the search activity is either to be informed or to see what is new or interesting. We found $13.4 \%$ search tasks that belong to this category, e.g. Keeping up-to-date with upcoming events. Users carry out serendipitous browsing to see if there are interesting events. "I was just trying to find out if there were any packages for dinner \& show or if there were any specials in restaurants, bars, clubs.. just to get an idea and see if I find something that stands out." [P7] 


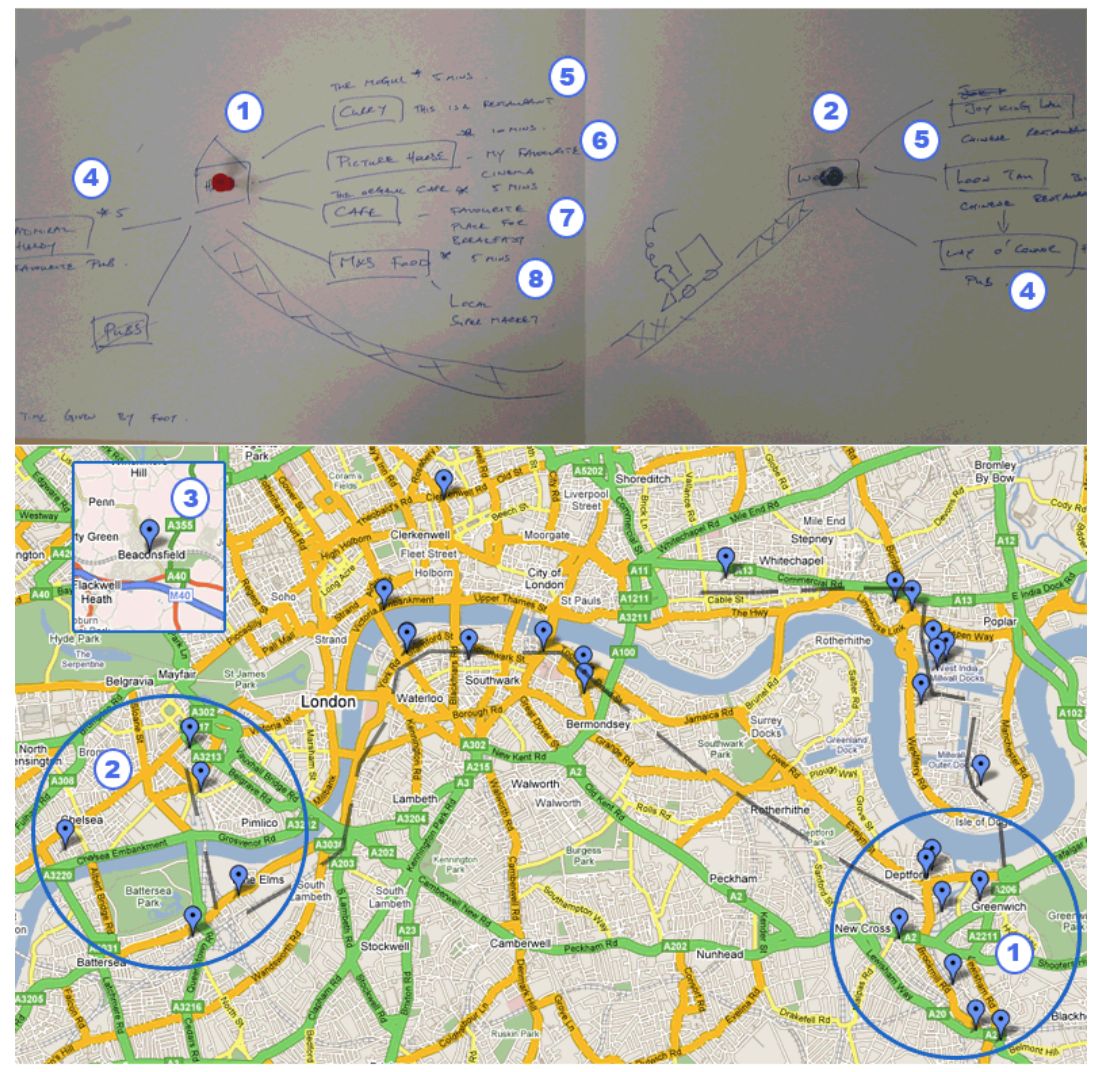

Fig. 2. Upper half: An example of a participant's sketch about his regular routes. Lower half: the related location tracking data. The markers represent approximate search locations. Multiple queries from one location are presented as one marker: (1) home area, (2) work area, (3) weekend holiday out of town, (4) pubs, (5) curry restaurant, (6) picture house cinema, (7) cafe, (8) supermarket. The dashed lines connecting areas (1) and (2) are the participant's daily train route.

\subsection{The Context of Location-Based Search}

The web-based diary tool gave us comprehensive information on where, when and how each location-based search occurs. We discuss each of these in the following subsections: spatial, temporal and social context.

\subsubsection{Spatial Context}

Participants' search activities and locations, while using the search engine, were logged throughout the study. We found that many searches are made either at home, at work, while travelling between the two, or at regularly visited places. With the exception of a weekend break, most of the time, participants' follow regularly used routes and regularly visited places (hotspots). Fig. 2 (lower half) shows a map visualization of searches made by a participant. Searches that have overlapping locations are 
represented by one point. The line connecting home (1) and work (2) represents a participant's daily travel routine with the train. At the post-study interview, we asked participants to make a sketch of the places they most frequently visit. For example, Fig. 2 (upper half) shows the corresponding sketches from the same participant. His regularly visited places are home, work, and several favourite businesses (pubs, restaurants, cinema, cafe) that are located within 5-15 minutes walking distance from home or work. By comparing the map visualization and the user sketches, we have a better understanding about our participants' spatial mobility, their hotspots, the distance between hotspots and their means of transport. From the 186 search tasks made by participants, the most common place to search was: at family/friends' home $(6.5 \%)$; public places $(8.5 \%)$, e.g. at the gym, at the pub/cafe; at work $(12 \%)$; on the move $(20 \%)$, e.g. on the public transport, inside a car; at home $(53 \%)$, e.g. watching $\mathrm{TV}$, lying in bed, preparing to go out. We discovered that the target location is more often related to their regularly visited places (e.g. work, home) rather than to the proximity of their current location, e.g. while on a way to work a participant searched for an optician close to home/work where he can easily stop by: "I needed to find the closest opticians and deals nearest to home or work." [P3].

\subsubsection{Temporal Context}

For every search task, participants were asked to specify whether the need for the information was spontaneous, related to something planned on that day, or arose in relation to something prior to that day. We found that more than half of the queries were a spontaneous need (66.1\% of 186 tasks), prompted mainly by activities and situations. They were based on recently acquired needs to obtain information and required immediate answers. "I needed to find a phone number for a client to contact him urgently." [P5] . Less than a quarter of the queries were needs that were planned for the same day but had less sense of urgency (21.5\% of 186 tasks), e.g. deciding where to go for lunch later. "Wanted to book a table for lunch with a local Tapas restaurant." [P1]. The remaining search tasks were needs for another day $(12.4 \%$ of 186 tasks). These tasks are mainly exploratory search and not urgent at all. "I was looking at a health farm for a weekend break." [P4]

\subsubsection{Social Context}

Participants were asked about who they were with at the moment they conducted the searches. More than three quarters of the location-based search tasks were conducted in the presence of others ( $76.1 \%$ of 186 tasks), e.g. while with family, friends or colleagues. Most location-based searches were prompted by: conversations with people, "We were talking about Rosarito and we wanted to know where that is." [P7]; event planning, "We were looking for somewhere to go for lunch on Easter Monday." [P5]; recommendation by people, "We have spoken about this wine tasting place and I wanted to go and have the experience." [P11]; and necessity, "We needed directions on how to get there." [P11]. Less than a quarter of location-based search tasks were conducted alone (23.9\% of 186 tasks), mostly driven by necessity. "I wanted to see that day's weather." [P5], "I wanted to know how to get from Hyde Park Corner to the City Airport." [P7].

Because of the common perception that a mobile device is a personal device, we were surprised to find most location-based searches are not merely a solitary activity 
but one that is strongly influenced and triggered by social interactions. Cui et al. [7] reported similar observation: that the mobile web acts as a conversation enhancer, such as to start a new topic, an ongoing discussion or settle a dispute. In our study, the mobile device plays a bigger role than merely as a communication tool and a conversation enhancer. Mobile search supports social activity, such as searching for ideas, collaborating on making plans, and sharing recommendations.

\subsubsection{Decision Making}

Related to the influence of spatial, temporal and social context, we investigated the decision making process in location-based search. In our post-study interview, 8-9 tasks were randomly chosen for every participant. For these tasks, participants were asked about the reason(s) behind choosing a certain business from the search result list. Table 2 shows the 9 most frequent reasons for choosing a certain place of interest. Most participants made the decision based on the availability of a specific product/service (23.7\%), e.g. checking if the store sells a particular product. "Needed to stock check a toy for my niece's $1^{\text {st }}$ birthday." [P1]. Many of the decision making processes in location-based searches were strongly influenced by social elements, such as recommended by other people (15.8\%) "Wanted to confirm the location of a pub. Had been given the name by a friend." [P1], or decided together with other people (13.2\%) e.g. "It's a restaurant to go for our anniversary." [P4], “

Table 2. Reasons to choose a place of interest from location-based search (Total: 76 tasks)

\begin{aligned} Reasons & \\ \hline $23.7 \% &$ has a particular product/service \\ $15.8 \% &$ is recommended by other people \\ $13.2 \% &$ is decided together * \\ $7.9 \% &$ is close to where I am now \\ $7.9 \% &$ It's my favorite place \\ $6.6 \% &$ is close to my home \\ $6.6 \% &$ is decided for work purpose \\ $5.3 \% &$ is decided by someone else \\ $3.9 \% &$ has the best offer (e.g. sale) \\ $9.1 \% &$ Other (e.g. serendipity search) \\ & * e.g. with friends, family, colleagues \end{aligned}

\subsection{Location-Based Information Sources}

Where participants were unable to satisfy their information need with the Google service, they were asked to report any other tools they used. A number of various locationbased information sources were used, such as: business directory, e.g. yell.com; event guide website, e.g. www.timeout.com; travel website, e.g. www.nationalrail.co.uk; news website, e.g. www.bbc.co.uk and information in public places, e.g. poster or map on the wall. Participants were also asked to note down if the search was successful or not. Of the 186 search tasks, $64.1 \%$ were successful, $24.3 \%$ were partially successful, and $11.7 \%$ were unsuccessful. Several reasons why location-based search on a mobile device was unsuccessful are, e.g. (a) Participant could not find local content or could not find up-to-date information. "Mainly American sites, couldn't find the UK one easily and then couldn't get the phone number." [P5]. "A very old wrestling information came 
up. Like years old." [P11]. (b) Difficult to conduct complex search with mobile interface. "National Rail Enquires needed the same information entering 2/3 times which was annoying and time consuming." [P1]. (c) Problems with mobile connection "It timed out a few times so I gave up." [P4], "Before I could finish my search, my train went into a tunnel and therefore no mobile connection." [P6]. When the search is unsuccessful, the participant has the choice to either abandon the search altogether, look for another information source, or develop a new problem solving strategy. "On Mobile, if I don't find something quickly then I give up, I lose interest. It would be rare to go back (to refine query). I guess it's cos you're used to going back using the back button on your PC ...on mobile you just give up." [P6], "There was nowhere I wanted to go in the search so I just decided to drive to the area and decide then." [P4].

\section{Discussion}

Our goal was to investigate location-based search needs and to understand the context in which they occurred. From the results, we distill a number of key findings:

Most location-based search is conducted in the presence of others. Although a mobile phone is a personal device, our study shows that more than three quarters of the searches are done in the presence of other people (76.1\% of 186 tasks). In some cases, these searches are group information needs rather than individual needs. This is because location-based mobile search is closely tied to social/group activities, such as going out. We also discovered that decision making in location-based search (e.g. where to go, which businesses to choose from) is highly influenced by social factors, such as recommended by people they know or decided together with family/friends.

Search now vs. search later. Our study reveals that mobile information seeking behavior progresses over time. A study on the mobile consumer behavior in 2001 reported that even though the information needs were triggered at a particular moment, people prefer to do Information Gathering search activities at a later time with their desktop, because of the difficulty in acquiring comprehensive information and making product comparison on the mobile phone [15]. In our study, this is partially still the case. Comparison search is still a difficult task to do on a mobile device. However, accessing information on the mobile device has become easier. On many occasions, participants prefer to use their mobile device at home rather than using their desktop because of the lower engagement threshold. For example, one participant and his daughter used the mobile search to plan their day while having breakfast in the kitchen. On another occasion he used mobile search just before bedtime while discussing weekend plans with his wife. These quick searches were considered inconvenient to do with the desktop computer located in the other room. Moreover, as they can now access many things on conveniently through the mobile device, there is less need to plan or to do search ahead of time. One participant puts it: "It will change a lot of things, you usually do your homework before (at home), now you can do it afterwards (on the road)."

Resident vs. Tourist. It should be noted that there are differences between the users in this study and the users in studies on mobile tourism, e.g. [6]. In our study, participants are residents, are somewhat familiar with the city, conduct location-based 
search regularly, and search on a broad domain of interest from plumbing to paint balling. Tourists, however, are more likely to be unfamiliar with the city (need to orient themselves often), have fewer time restrictions, and search for specific domains of interest e.g. city landmarks and how to get to these places [6]. We expect differences between the two user roles with respect to location-based search types, and their spatial, temporal, and social context.

Study limitation. We acknowledge that there are several limitations to our study. First, our participants are Blackberry users. As pointed out in [9], users with Qwerty keyboards may have different search patterns compared to users with 12-keypad devices with regards to the distribution of number of words per query, domain of interest distribution, query distribution and session characteristics. Nevertheless, we expect users' location-based information needs and the aspects investigated in this study (information seeking tasks, the role of spatial, temporal and social context) are mobile device independent. Second, our study had a relatively homogeneous demographic profile. This should not come as a surprise. The reality is that smart phone and mobile Web penetration is highest amongst a fairly narrow group who tend to be young professionals. It would not have been logical to recruit a cross section of all mobile users since we wanted to ensure we only recruited people who already use and were thus familiar with mobile search. It should be acknowledged that the findings of this research may vary for different user segment. However, this research is among the first to report on location-based search actual usage.

\section{Design Implications}

Location-based search experience can be improved by taking into account users' motivation, search patterns and context. We present a list of issues that should be addressed in future system designs.

Detecting and predicting location-based information needs. As mentioned in [22], it is difficult for a search engine to identify whether a query is location-based without any location qualifier. In our study, we found that most of the time participants only specify business name, business category, event name or product name. Thus, based on our findings we recommend that whenever a search engine receives a query that contains one of these elements, the search engine should prioritize using locationbased information.

Recommendation based on hotspots. We found that people move along regularly used routes and their location-based search interests are within their hotspots (as shown in Fig. 2). Moreover, users' interests in location-based information are usually within the proximity of their hotspots. Thus, a personalized search result that provides location-based information tailored to users' hotspots areas would be potentially valuable.

Location-based search query refinement. Features that help avoid making mistakes when typing unfamiliar business/place names and addresses is useful to have in search engines. Search engines can help users by providing query suggestions based on likely local business/place names, for example, a list of query refinements, similar 
to the 'Did you Mean?' feature for business/place names that are phonetically or morphologically similar to what has been typed. Another useful feature to help users specify business names and places is to provide autocompletion suggestions of businesses nearby or favorite businesses from users' bookmark or search history.

Support iterative, exploratory and comparative search activity. Our results show that a large portion of our search tasks are Information Gathering tasks (43.8\%, see Fig. 1B). Most search interfaces, however, do not provide features that support iterative, exploratory, and comparative search activities. Features that would help users in this task are: first, support users to collect, filter, organize, compare, save and share location-based search results; second, support exploration by allowing users to tweak a set of constraints, such as enable to filter points of interest by distance, business category, service price, in order to find the optimum search results.

Location-based services mash-ups. Integration of different services, such as business directory (restaurant, pub, store), places (car parks, bus stops), public transport system (train, bus, tube), navigation system (route, distance), other services (ATM, gas station, restroom) and map application is another way to help users in Information Gathering tasks. Integration of different services will help them decide where to go, match their schedules, estimate distance and travel time between places, and ultimately help users make better plans.

Recommendation based on social network. Recommendations made by people play an important role in prompting location-based search needs and the decision making processes. It would be helpful to provide several features: (a) enable people to search, recommend and share experiences on businesses and make this information easily accessible to people from their social networks; (b) to provide location-based recommendations based on the interests of the whole group, for example if people within a social network are detected to be in close proximity to each other, a search engine can recommend places of interest that incorporate the interests of all members of the group rather than just the searcher.

\section{Conclusion and Future Work}

We conducted an in-depth web-based diary study using different types of data collected from search event logs, location tracking and diary entries. We found that location-based searches are mostly based upon just-in-time information needs that are usually related to social activity. Our study also shows that participants have regularly used routes and regularly visited places (hotspots). We also found that the target locations for these searches are more often related to users' regularly visited places (e.g. work, home). Services that support location-based search need to take user's social and spatial context into account. There is still much work to be done on how to properly implement search recommendations based on users' hotspots and social networks, e.g. with respect to information privacy and security. Finally, as mobile web searching becomes more widely adopted, we believe that location-based search will be an even more prominent need that calls for alternative mobile search interfaces and new engaging ways to interact with location-based information. 
Acknowledgments. We thank Google for the opportunity to conduct this research; Liviu Tancau and Terry Van Belle for the exceptional technical support; Jens Riegelsberger and Robin Jeffries for the feedback on a draft of this paper.

\section{References}

1. Amin, A., Hardman, L., van Ossenbruggen, J., van Nispen. A.: Understanding cultural heritage experts' information seeking tasks. In: Proc. JCDL 2008, pp. 39-47 (2008)

2. Baeza-Yates, R., Dupret, G.: Velasco. J.: A study of mobile search queries in Japan. In: Query Log Analysis: Social and Technological Challenges, at WWW 2007 (2007)

3. Brandt, J., Weiss, N., Klemmer, S.R.: txt 4 18r: lowering the burden for diary studies under mobile conditions. In: Proc. CHI 2007, pp. 2303-2308 (2007)

4. Broder, A.: A taxonomy of web search. SIGIR Forum 36(2), 3-10 (2002)

5. Church, K., Smyth, B., Cotter, P., Bradley, K.: Mobile information access: A study of emerging search behavior on the mobile internet. ACM Trans. Web 1(1), 4 (2007)

6. Brown, B., Chalmers, M.: Tourism and mobile technology. In: Proc. ECSCW 2003, pp. 335-354. Kluwer Academic Publishers, Dordrecht (2003)

7. Consolvo, S., Walker, M.: Using the experience sampling method to evaluate ubicomp applications. IEEE Pervasive Computing 2(2), 24-31 (2003)

8. Cui, Y.: Roto. V.: How people use the web on mobile devices. In: Proc. WWW 2008, pp. 905-914 (2008)

9. Kamvar, M.: Baluja. S.: A large scale study of wireless search behavior: Google mobile search. In: Proc. CHI 2006, pp. 701-709 (2006)

10. Kamvar, M., Baluja, S.: The Role of Context in Query Input: Using contextual signals to complete queries on mobile devices. In: Proc. MobileHCI 2007 (2007)

11. Kellar, M., Watters, C., Shepherd, M.: A field study characterizing web-based information seeking tasks. Technical report, Dalhousie University Halifax (2005)

12. Kellar, M., Watters, C., Shepherd, M.: The impact of task on the usage of web browser navigation mechanisms. In: Proc. GI 2006, pp. 235-242. Canadian Information Processing Society (2006)

13. Lynch, K.: The image of the city. The MIT Press, Cambridge (1960)

14. Mark, G., Christensen, U., Shafae, M.: A methodology using a microcamera for studying mobile. In: IT Usage and Person Mobility, CHI 2001 Workshop on Mobile Communications: Understanding Users, Adoption \& Design (2001)

15. O'Hara, K., Perry, M.: Shopping anytime anywhere. In: Proc. CHI 2001, pp. 345-346 (2001)

16. Palen, L., Salzman, M.: Voice-mail diary studies for naturalistic data capture under mobile conditions. In: Proc. CSCW 2002, pp. 87-95 (2002)

17. Riegelsberger, J., Nakhimovsky, Y.: Seeing the bigger picture: a multi-method field trial of google maps for mobile. In: Proc. CHI 2008, pp. 2221-2228 (2008)

18. Sanderson, M., Han, Y.: Search words and geography. In: Proc. GIR 2007, pp. 13-14 (2007)

19. Sanderson, M., Kohler, J.: Analyzing geographic queries. In: Workshop on Geographic Information Retrieval SIGIR (2004)

20. Sohn, T., Li, K.A., Griswold, W.G., Hollan, J.D.: A diary study of mobile information needs. In: Proc. CHI 2008, pp. 433-442 (2008)

21. Spink, A., Jansen, B., Wolfram, D., Saracevic, T.: From E-Sex to E-Commerce: Web Search Changes, pp. 107-109. IEEE Computer Society, Los Alamitos (2002)

22. Yi, J., Maghoul, F.: Pedersen. J.: Deciphering mobile search patterns: a study of yahoo! mobile search queries. In: Proc. WWW 2008, pp. 257-266 (2008) 